\title{
Correlation between tobacco control policies and mortality of haematological cancers across Europe: An ecological study
}

\author{
Dipal Mehta ${ }^{1+}$, Rory Dennis ${ }^{2+}$, Susanna Nallamilli ${ }^{3}$, Mathew Vithayathil ${ }^{4,5,6}$, Jose María Martínez-Sánchez ${ }^{5,7}$
}

\begin{abstract}
INTRODUction To determine the correlation between tobacco control policies and mortality of haematological malignancies: leukemia, lymphoma and multiple myeloma (MM).

METHODS Ecological study with the countries as the unit of analysis. Tobacco Control Scale (TCS) scores from 2010, 2013 and 2016 were used as measures for the level of tobacco control policy implementation in 27 European countries. Mortality rates for leukemia, lymphoma, and MM, were obtained from the WHO Mortality Database and the European Cancer Information System for each country for 2010, 2013, 2015 and 2018. Correlation between yearly TCS scores and mortality rates from the same and prospective years were calculated using Spearman's rank correlation coefficients $\left(\mathrm{r}_{\mathrm{sp}}\right)$ and 95\% confidence intervals (95\% CI) (2010 TCS scores vs 2010, 2013, 2015, 2018 mortality rates; 2013 TCS scores vs 2013, 2015, 2018 mortality rates; and 2016 TCS scores vs 2018 mortality rates).

RESULTS The 2010 TCS scores were significantly negatively associated with leukemia mortality rates in $2013\left(\mathrm{r}_{\mathrm{sp}}=-0.58 ; 95 \%\right.$ CI: $\left.-0.79,-0.24 ; \mathrm{p}=0.002\right)$, $2015\left(\mathrm{r}_{\mathrm{sp}}=-0.65 ; 95 \%\right.$ CI: $\left.-0.85,-0.30 ; \mathrm{p}=0.001\right)$ and $2018\left(\mathrm{r}_{\mathrm{sp}}=-0.44 ; 95 \%\right.$ CI: $-0.71,-0.06 ; \mathrm{p}=0.021)$. TCS scores from 2013 and 2016 had significant negative associations with leukemia mortality in all prospective years. TCS scores did not demonstrate consistent correlations with lymphoma and MM mortality.

CONCLUSIONS The level of tobacco control policies in European countries correlates negatively with leukemia mortality at ecological level, with no correlation seen for lymphoma and MM. This study advocates that increased tobacco control implementation may improve leukemia mortality.
\end{abstract}

\section{AFFILIATION \\ 1 Department of Haematology, Hammersmith Hospital, Imperial College Healthcare NHS Trust, London, United Kingdom \\ 2 Department of Anaesthetics, Mid Essex NHS Foundation Trust, Essex, United Kingdom \\ 3 Department of Medicine, King's College Hospital NHS Foundation Trust, London, United Kingdom 4 Department of Gastroenterology, Imperial College Healthcare NHS \\ Trust, London, United Kingdom \\ 5 Department of Epidemiology, Harvard T. H. Chan School of Public Health, Boston, United States 6 Department of Nutrition, Harvard T. H. Chan School of Public Health, Boston, United States \\ 7 Group of Evaluation of Health Determinants and Health Policies, Universitat Internacional de Catalunya, Barcelona, Spain + Co-first authors}

\section{CORRESPONDENCE TO}

Mathew Vithayathil. Department of Gastroenterology, Imperial College Healthcare NHS Trust, St Mary's Hospital, South Wharf Road, W2 1NY, London, United Kingdom. E-mail: mathew.vithayathil@ doctors.org.uk

\section{KEYWORDS}

cancer, multiple myeloma, leukemia, tobacco control, Europe, non-Hodgkin lymphoma

Received: 18 September 2020 Revised: 22 January 2021

Accepted: 1 February 2021

\section{INTRODUCTION}

Tobacco-related mortality is the highest cause of preventable death worldwide ${ }^{1}$, with both direct and secondhand smoking ( $\mathrm{SHS}$ ) causing significant harm ${ }^{2,3}$. Cigarette smoking is a well-established carcinogen for humans, and multiple cancers including lung, head and neck, upper digestive tract, kidney, and bladder, are well known for their strong 
association with smoking ${ }^{4}$.

The role of smoking as a risk factor for the development of haematological malignancies is established. Both adult and parental smoking are risk factors for adult ${ }^{5}$ and childhood leukemias ${ }^{6}$. However, the role smoking plays in lymphoma and multiple myeloma (MM) is less clear, with inconsistent associations observed ${ }^{7,8}$.

Previous ecological studies have demonstrated national tobacco control policies have impacts on smoking prevalence ${ }^{9,10}$, tobacco consumption and cessation $^{11,12}$, SHS exposure ${ }^{10}$, and attitudes towards smoking regulations ${ }^{10,13}$. The effects of tobacco control on disease have also been demonstrated, with the implementation of stronger policies associated with lower rates of lung cancer ${ }^{14}$ and preterm births ${ }^{15}$.

We aimed to assess the correlation between the implementation of tobacco control policies and mortality from haematological cancers across 27 European countries.

\section{METHODS}

This is an ecological study with each country as the unit of analysis. The 27 European countries included were: Austria, Belgium, Bulgaria, Cyprus, Czech Republic, Denmark, Estonia, Finland, France, Germany, Greece, Hungary, Ireland, Italy, Latvia, Lithuania, Luxembourg, Malta, Netherlands, Poland, Portugal, Romania, Slovakia, Slovenia, Spain, Sweden, and the United Kingdom.

The degree of tobacco control policies for each European country was measured using the Tobacco Control Scale (TCS) ${ }^{16,17}$. The TCS is a tool developed in 2006 to quantify the implementation of strategies directed at reducing tobacco use at the country level. The scale allocates points (maximum 100) for implementation of six key policies including: taxation of tobacco products, bans/restrictions of public and workplace smoking, bans on smoking advertising, improvement of the information provider to tobacco consumers, adoption of health warnings of tobacco product boxes, and smoking cessation treatments. TCS scores for the years 2010, 2013 and 2016 were obtained for the 27 European countries.

Mortality data were calculated for leukemia, lymphoma and MM across the 27 European countries. Rates for 2010, 2013 and 2015 were obtained from the World Health Organization (WHO) Mortality Database ${ }^{18}$. Age-standardized rate (ASR) was calculated using the WHO standard population ${ }^{19}$. For 2018 , mortality data were obtained from the European Cancer Information System (ECIS) website ${ }^{20}$. Estimated mortality data were calculated from the International Agency for Research on Cancer (IARC) using historical data on incidence and mortality from the WHO Mortality Database $^{21}$. ASR was obtained for leukemia and MM, with crude mortality rates used for lymphoma.

We calculated Spearman's rank correlation coefficients $\left(\mathrm{r}_{\mathrm{sp}}\right)$ and $95 \%$ confidence intervals $(95 \%$ CI) between scores in the TCS for 2010, 2013 and 2016 and mortality rates for haematological cancers for 2010, 2013, 2015 and 2018 for the 27 countries. TCS scores for each year were correlated with mortality rates from the same and prospective years (i.e. TCS scores from 2010 were analyzed with mortality data from 2010, 2013, 2015 and 2018; TCS scores from 2013 were analyzed with mortality data from 2013, 2015 and 2018; and TCS scores from 2016 were only analyzed with mortality data 2018). For leukemia, we conducted linear regression between age-standardized mortality (ASM) and TCS score year by year. Beta coefficients representing change in ASM per 10-point increase in TCS score were calculated with $95 \%$ confidence intervals.

\section{RESULTS}

Table 1 shows the year-by-year correlations between TCS scores and mortality rates for each haematological cancer. TCS scores ranged from 32 to 88 . Ranges for age-standardized mortality rates (per 100000 persons) were 2.4-5.9 for leukemia, 2.23.7 for lymphoma and 0.5-2.4 for MM. Significant negative correlations were demonstrated between TCS scores and leukemia mortality. TCS scores from 2010 showed significant negative correlation with leukemia mortality in $2013\left(\mathrm{r}_{\mathrm{sp}}=-0.58 ; 95 \%\right.$ CI: $-0.79,-0.24 ; p=0.002), 2015\left(r_{\mathrm{sp}}=-0.65 ; 95 \%\right.$ CI: $-0.85,-0.30 ; \mathrm{p}=0.001)$ and $2018\left(\mathrm{r}_{\mathrm{sp}}=-0.44 ; 95 \% \mathrm{CI}\right.$ : $-0.71,-0.06 ; p=0.021)$. Similarly, significant negative associations were observed for 2013 TCS scores and leukemia mortality for $2013\left(\mathrm{r}_{\mathrm{sp}}=-0.58 ; 95 \% \mathrm{CI}\right.$ : $-0.79,-0.24 ; \mathrm{p}=0.002), 2015\left(\mathrm{r}_{\mathrm{sp}}=-0.51 ; 95 \% \mathrm{CI}\right.$ : $-0.77,-0.10 ; \mathrm{p}=0.002)$ and $2018\left(\mathrm{r}_{\mathrm{sp}}=-0.50 ; 95 \% \mathrm{CI}\right.$ : $-0.75,-0.14 ; \mathrm{p}=0.010)$; and between 2016 TCS scores 
Table 1. Spearman correlation coefficients $\left(r_{s p}\right)$ and $95 \%$ confidence interval between Tobacco Control Scale (TCS) scores $(2010,2013,2016)^{a}$ and mortality rates $(2010,2013,2015,2018)$ for leukemia, lymphoma and multiple myeloma in 27 European countries (2010 TCS scores vs 2010, 2013, 2015, 2018 mortality rates; 2013 TCS scores vs 2013, 2015, 2018 mortality rates; 2016 TCS scores vs 2018 mortality rates)

\begin{tabular}{|c|c|c|c|c|}
\hline Malignancies & $2010^{b}$ & $2013^{b}$ & $2015^{b}$ & $2018^{c}$ \\
\hline \multicolumn{5}{|l|}{ Leukemia } \\
\hline TCS 2010 & $\begin{array}{c}-0.35(-0.65,0.04) \\
p=0.070\end{array}$ & $\begin{array}{c}-0.58(-0.79,-0.24) \\
p=0.002\end{array}$ & $\begin{array}{c}-0.65(-0.85,-0.30) \\
p=0.001\end{array}$ & $\begin{array}{c}-0.44(-0.71,-0.06) \\
p=0.021\end{array}$ \\
\hline TCS 2013 & - & $\begin{array}{c}-0.58(-0.79,-0.24) \\
p=0.002\end{array}$ & $\begin{array}{c}-0.51(-0.77,-0.10) \\
p=0.002\end{array}$ & $\begin{array}{c}-0.50(-0.75,-0.14) \\
p=0.010\end{array}$ \\
\hline TCS 2016 & - & - & - & $\begin{array}{c}-0.40(-0.68,-0.01) \\
p=0.040\end{array}$ \\
\hline \multicolumn{5}{|l|}{ Lymphoma } \\
\hline TCS 2010 & $\begin{array}{c}0.36(-0.06,0.67) \\
p=0.080\end{array}$ & $\begin{array}{c}0.48(0.10,0.74) \\
p=0.014\end{array}$ & $\begin{array}{c}0.35(-0.11,0.68) \\
p=0.123\end{array}$ & $\begin{array}{c}0.33(-0.07,0.64) \\
p=0.089\end{array}$ \\
\hline TCS 2013 & - & $\begin{array}{c}0.28(-0.14,0.61) \\
p=0.170\end{array}$ & $\begin{array}{c}0.19(-0.27,0.59) \\
p=0.399\end{array}$ & $\begin{array}{c}0.09(-0.34,0.46) \\
p=0.669\end{array}$ \\
\hline TCS 2016 & - & - & - & $\begin{array}{c}0.09(-0.31,0.47) \\
p=0.650\end{array}$ \\
\hline \multicolumn{5}{|c|}{ Multiple myeloma } \\
\hline TCS 2010 & $\begin{array}{c}0.29(-0.14,0.62) \\
p=0.166\end{array}$ & $\begin{array}{c}0.47(-0.08,0.73) \\
p=0.016\end{array}$ & $\begin{array}{c}0.10(-0.36,0.52) \\
p=0.657\end{array}$ & $\begin{array}{c}0.14(-0.26,0.50) \\
p=0.479\end{array}$ \\
\hline TCS 2013 & - & $\begin{array}{c}0.20(-0.21,0.56) \\
p=0.320\end{array}$ & $\begin{array}{c}-0.05(-0.48,0.40) \\
p=0.825\end{array}$ & $\begin{array}{c}-0.12(0.49,0.28) \\
p=0.560\end{array}$ \\
\hline TCS 2016 & - & - & - & $\begin{array}{c}-0.05(-0.43,0.35) \\
p=0.806\end{array}$ \\
\hline
\end{tabular}

a Tobacco Control Scale (TCS) scores and age standardized mortality rates obtained from 27 European countries (Austria, Belgium, Bulgaria, Cyprus, Czech Republic, Denmark, Estonia, Finland, France, Germany, Greece, Hungary, Ireland, Italy, Latvia, Lithuania, Luxembourg, Malta, Netherlands, Poland, Portugal, Romania, Slovakia, Slovenia, Spain, Sweden and the United Kingdom). b Definitive age standardized mortality rates for 2010, 2013 and 2015 obtained from WHO Mortality Database ${ }^{18}$. c Estimated mortality rates for 2018 from European Cancer Information System. Age standardized rates were obtained for leukemia and multiple myeloma; crude mortality rates were obtained for lymphoma ${ }^{20}$.

Figure 1. Linear regression beta coefficients and 95\% confidence interval (95\% CI) representing change in leukemia age-standardised mortality rates $(2010,2013,2015,2018)$ per 10-point increase in Tobacco Control Scale (TCS) score $(2010,2013,2016)$ in 27 European countries

TCS 2010
Mortality 2010

Mortality 2013

Mortality 2015

Mortality 2018

TCS 2013

Mortality 2013

Mortality 2015

Mortality 2018

TCS 2016

Mortality 2018

$-0.8$
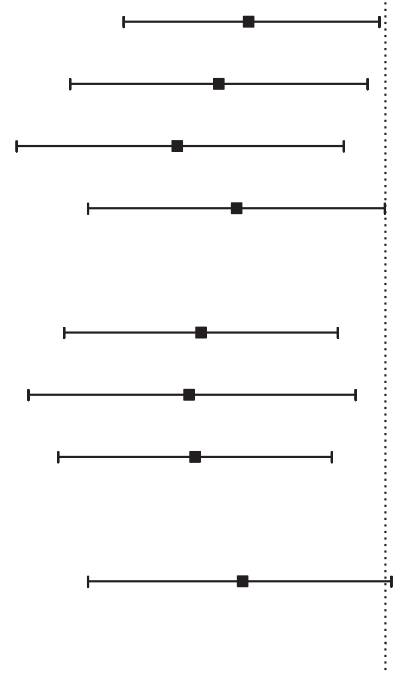

$-0.28(-0.53,-0.03), p=.028$

$-0.35(-0.62,-0.07), p=.016$

$-0.25(-0.50,-0.001), p=.049$

$-0.31(-0.54,-0.08), p=.010$

$-0.33(-0.60,-0.05), p=.021$

$-0.32(-0.55,-0.09), p=.007$

$-0.24(-0.50,0.01), p=.059$

$\begin{array}{lll}0.2 & 0.4 & 0.6\end{array}$
$-0.23(-0.44,-0.01), p=.041$

Change in age-standardised mortality rate of leukaemia $(95 \% \mathrm{Cl})$ per 10-point increase in TCS Score 
and 2018 leukemia mortality rates $\left(\mathrm{r}_{\mathrm{sp}}=-0.40 ; 95 \%\right.$ CI: -0.68, -0.01; $\mathrm{p}=0.040)$. No consistent significant correlations were observed between TCS scores and lymphoma and MM mortality rates. In further analysis, we conducted linear regression for TCS scores and age-standardized leukemia mortality. Estimates for changes in leukemia ASM per 10-point increase in TCS scores are shown in Figure 1. Consistent negative beta coefficients were seen (range: $-0.24,-0.35$ ), with significant decreases seen between TCS scores from 2010 and 2013 and leukemia mortality in 2010, 2013, 2015 and 2018.

\section{DISCUSSION}

This is the first study to establish the correlation between tobacco control policies and haematological malignancy in Europe. We observe increasing tobacco control measures have a negative association with mortality from leukemia, with significant decreases in mortality seen per 10-point increase in TCS score. In contrast, we observed no consistent association between tobacco control measures and lymphoma and MM mortality.

Tobacco control policies lead to a reduction in tobacco usage. Two studies across 27 European countries have demonstrated that increased TCS scores were associated with decreased smoking prevalence and high rates of smoking cessation and intention to quit ${ }^{11,12}$, with reduced rates of smoking and SHS observed longitudinally as well ${ }^{22}$. With the established role of smoking as a risk factor for leukemia, it stands to reason that increased TCS scores will lead to a decrease in leukemia mortality.

Acute myeloid leukemia (AML) is the most common acute leukemia and is also the most strongly linked with smoking ${ }^{23}$. A recent metaanalysis demonstrated a $40 \%$ increase in the risk of AML in current smokers, and a $27 \%$ increase in AML risk in ever smokers ${ }^{5}$. The same metaanalysis also showed a direct association between intensity and duration of smoking and the risk of AML. Moreover, recent data from a Danish National Leukemia Registry revealed that smoking status was associated with an inferior overall survival in patients with AML who underwent intensive treatment ${ }^{24}$. Acute lymphoblastic leukemia (ALL) is rare in the adult population in comparison to $\mathrm{AML}$, whereas it accounts for approximately $80 \%$ of childhood leukemia diagnoses ${ }^{25}$. As such, there are few data on smoking and the risk of adult-onset ALL. However, a recent meta-analysis has demonstrated an association between paternal smoking both pre-conception and during pregnancy, and subsequent development of childhood ALL.

There are multiple biological mechanisms to account for this link. Cigarette smoking exposes the bone marrow to benzene, which is a potent carcinogen associated with leukemogenesis ${ }^{26}$. Furthermore, smoking has been shown to reduce the number of circulating CD34+ progenitor cells, a key element of the haematopoetic system ${ }^{27}$.

Whilst multiple mechanisms exist to explain the link between smoking and leukemia, there remains a lack of clarity regarding the time lag between smoking exposure and leukemia development. This is difficult to establish as the development of acute leukemia follows a step-wise process where a combination of mutations or lesions leads to an expansion of a sub-clone of neoplastic cells after a variable latency period ${ }^{28}$. Nonetheless, studies have attempted to establish temporal associations between smoking exposure and leukemia development. A population-based case-control study in Germany ${ }^{29}$ found a positive trend in the development of acute non-lymphocytic leukemia in patients with smoking exposure in the prior 2 to 10 years before diagnosis, with no such trend identified for smoking exposure in the prior 10 to 20 years before diagnosis. Furthermore, pre-conception paternal smoking is associated with childhood ALL up to 5 years old ${ }^{30}$. The timeframe of our study is in line with this latency period and would therefore capture the effect of changing tobacco control policies on leukemia mortality.

Studies investigating the role of tobacco in lymphoma and MM development are inconsistent. Non-Hodgkin's lymphoma (NHL) accounts for the majority of lymphoma diagnoses, with multiple histopathological subtypes with varying disease courses. Due to the heterogenous nature of NHL, conclusions regarding smoking as a risk factor are difficult to interpret. Though the subtype follicular lymphoma demonstrates an association with smoking, more prominent in females, a lack of consistent association was seen in other subtypes ${ }^{31}$. MM is a plasma cell malignancy where the aetiology 
is yet to be fully established. Cigarette smoking has not been implicated as a risk factor, with studies such as a recent pooled analysis led by the Multiple Myeloma Consortium failing to show any association $^{32}$. We observed significant associations between mortality for lymphoma and MM in 2013 and TCS scores in 2013, but no consistent correlations between tobacco control and lymphoma and MM related mortality, consistent with previous studies.

\section{Limitations}

The main limitation of the study is the ecological design. Correlations observed could be due to confounding factors, demonstrated to be risk factors for haematological malignancy, such as socioeconomic status $^{33}$, alcohol intake ${ }^{34}$, and access to cancer therapy. There may be a wide range of overlapping factors affecting the effectiveness of tobacco control policies and public health policies related to haematological malignancy. These may range from contextual factors, such as national socioeconomic and demographic distributions, to general policy approaches to education and equity. More specific factors, including fiscal policies (for instance taxation affecting smoking and alcohol purchasing) and healthcare system approaches (affecting both tobacco cessation and cancer prevention and treatment) may also contribute to confounding. Moreover, the ecological study does not allow us to extrapolate the associations found at the country level to individuals (causality). Similarly, cancer mortality rates for 2018 were obtained from estimates from historical data, and potentially inaccurate. However, correlations observed for 2018 mortality rates were consistent with other years.

\section{CONCLUSIONS}

This study demonstrates, at the ecological level, that tobacco control is inversely correlated with leukemia mortality. There was no consistent association between tobacco control and lymphoma or multiple myeloma mortality. These findings advocate the implementation of public health strategies to increase tobacco control policies to reduce leukemia mortality.

\section{REFERENCES}

1. Jha P. Avoidable global cancer deaths and total deaths from smoking. Nat Rev Cancer. 2009;9(9):655-64. doi:10.1038/nrc2703

2. Öberg M, Jaakkola MS, Woodward A, Peruga A, PrüssUstün A. Worldwide burden of disease from exposure to second-hand smoke: a retrospective analysis of data from 192 countries. Lancet. 2011;377(9760):139-146. doi:10.1016/S0140-6736(10)61388-8

3. U.S. Department of Health and Human Services. The Health Consequences of Smoking: 50 Years of Progress. A Report of the Surgeon General. Atlanta, GA: U.S. Department of Health and Human Services, Centers for Disease Control and Prevention, National Center for Chronic Disease Prevention and Health Promotion, Office on Smoking and Health; 2014. Accessed September 18, 2020. https://www.ncbi.nlm.nih.gov/books/ NBK179276/pdf/Bookshelf_NBK179276.pdf

4. Vineis P, Alavanja M, Buffler P, et al. Tobacco and cancer: recent epidemiological evidence. J Natl Cancer Inst. 2004;96(2):99-106. doi:10.1093/jnci/djh014

5. Fircanis S, Merriam P, Khan N, Castillo JJ. The relation between cigarette smoking and risk of acute myeloid leukemia: an updated meta-analysis of epidemiological studies. Am J Hematol. 2014;89(8):E125-132. doi:10.1002/ajh.23744

6. Cao Y, Lu J, Lu J. Paternal Smoking Before Conception and During Pregnancy Is Associated With an Increased Risk of Childhood Acute Lymphoblastic Leukemia: A Systematic Review and Meta-Analysis of 17 Case-Control Studies. J Pediatr Hematol Oncol. 2020;42(1):32-40. doi:10.1097/MPH.0000000000001657

7. Lu Y, Wang SS, Reynolds P, et al. Cigarette smoking, passive smoking, and non-Hodgkin lymphoma risk: evidence from the California Teachers Study. Am J Epidemiol. 2011;174(5):563-573. doi:10.1093/aje/kwr127

8. Ugai T, Ito H, Oze I, et al. Association of BMI, Smoking, and Alcohol with Multiple Myeloma Mortality in Asians: A Pooled Analysis of More than 800,000 Participants in the Asia Cohort Consortium. Cancer Epidemiol Biomarkers Prev. 2019;28(11):1861-1867. doi:10.1158/1055-9965.EPI-19-0389

9. Gallus S, Lugo A, La Vecchia C, et al. Pricing Policies And Control of Tobacco in Europe (PPACTE) project: cross-national comparison of smoking prevalence in 18 European countries. Eur J Cancer Prev. 2014;23(3):177185. doi:10.1097/CEJ.0000000000000009

10. Martínez-Sánchez JM, Fernández E, Fu M, et al. Smoking behaviour, involuntary smoking, attitudes towards smoke-free legislations, and tobacco control activities in the European Union. PLoS One. 2010;5(11):e13881. doi:10.1371/journal.pone.0013881

11. Feliu A, Filippidis FT, Joossens L, et al. Impact of tobacco control policies on smoking prevalence and quit ratios in 27 European Union countries from 2006 to 2014. Tob Control. 2019;28(1):101-109. doi:10.1136/tobaccocontrol-2017-054119

12. Lidón-Moyano G, Martín-Sánchez JC, Saliba P, 
Graffelman J, Martínez-Sánchez JM. Correlation between tobacco control policies, consumption of rolled tobacco and e-cigarettes, and intention to quit conventional tobacco, in Europe. Tob Control. 2017;26(2):149-152. doi:10.1136/tobaccocontrol-2015-052482

13. Lidón-Moyano C, Fu M, Perez-Ortuño R, et al. Assessment of salivary cotinine concentration among general nonsmokers population: Before and after Spanish smoking legislations. Cancer Epidemiol. 2017;51:87-91. doi:10.1016/j.canep.2017.10.011

14. González-Marrón A, Martín-Sánchez JC, Miró Q, MatillaSantander N, Cartanyà-Hueso À, Mucci L, MartínezSánchez JM. Relation between tobacco control policies and population at high risk of lung cancer in the European Union. Environ Res. 2019;179(pt A):108594. doi:10.1016/j.envres.2019.108594

15. Díez-Izquierdo A, Balaguer A, Lidón-Moyano C, MartínSánchez JC, Galán I, Fernández E, Martínez-Sánchez JM. Correlation between tobacco control policies and preterm births and low birth weight in Europe. Environ Res. 2018;160:547-553. doi:10.1016/j.envres.2017.10.033

16. Joossens L, Raw M. The Tobacco Control Scale: a new scale to measure country activity. Tob Control. 2006;15(3):247-253. doi:10.1136/tc.2005.015347

17. Methods. Tobacco Control Scale Website. Accessed September 18, 2020. https://www.tobaccocontrolscale. org/methods/

18. World Health Organization. WHO Mortality Database. Updated March 1, 2021. Accessed March 2, 2020. https://www.who.int/ data/data-collection-tools/who-mortality-database

19. Ahmad OB, Boschi-Pinto C, Lopez AD, Murray CJL, Lozano R, Inoue M. Age standardization of rates: a new WHO standard. World Health Organization; 2001. GPE Discussion Paper Series, No.31. Accessed September 18, 2020. https://www.researchgate.net/ publication/203609941_Age_Standardization_of_ Rates_A_New_WHO_Standard

20. European Commission. ECIS - European Cancer Information System. Accessed March 2, 2020. https:// ecis.jrc.ec.europa.eu/

21. Ferlay J, Colombet M, Soerjomataram I, et al. Estimating the global cancer incidence and mortality in 2018: GLOBOCAN sources and methods. Int J Cancer. 2019;144(8):1941-1953. doi:10.1002/ijc.31937

22. Olivieri M, Murgia N, Carsin AE, et al. Effects of smoking bans on passive smoking exposure at work and at home. The European Community respiratory health survey. Indoor Air. 2019;29(4):670-679. doi:10.1111/ina.12556

23. Chelghoum Y, Danaïla C, Belhabri A, et al. Influence of cigarette smoking on the presentation and course of acute myeloid leukemia. Ann Oncol. 2002;13(10):1621-1627. doi:10.1093/annonc/mdf269

24. Kristensen D, Nielsen LB, Roug AS, et al. The prognostic effect of smoking status on intensively treated acute myeloid leukaemia - A Danish nationwide cohort study. Br
J Haematol. 2020;190(2):236-243. doi:10.1111/bjh.16667

25. Ward E, DeSantis C, Robbins A, Kohler B, Jemal A. Childhood and adolescent cancer statistics, 2014. CA Cancer J Clin. 2014;64(2):83-103. doi:10.3322/caac.21219

26. Snyder R. Leukemia and benzene. Int J Environ Res Public Health. 2012;9(8):2875-2893. doi:10.3390/ijerph9082875

27. Ludwig A, Jochmann N, Kertesz A, et al. Smoking decreases the level of circulating CD34+ progenitor cells in young healthy women-a pilot study. BMC Womens Health. 2010;10:20. doi:10.1186/1472-6874-10-20

28. Valent P, Kern W, Hoermann G, et al. Clonal Hematopoiesis with Oncogenic Potential (CHOP): Separation from CHIP and Roads to AML. Int J Mol Sci. 2019;20(3):789. doi:10.3390/ijms20030789

29. Richardson DB, Terschüren C, Pohlabeln H, Jöckel KH, Hoffmann W. Temporal patterns of association between cigarette smoking and leukemia risk. Cancer Causes Control. 2008;19(1):43-50. doi:10.1007/s10552-007-9068-7

30. Ji BT, Shu XO, Linet MS, Zheng W, Wacholder S, Gao YT, Ying DM, Jin F. Paternal cigarette smoking and the risk of childhood cancer among offspring of nonsmoking mothers. J Natl Cancer Inst. 1997;89(3):238-244. doi:10.1093/jnci/89.3.238

31. Linet MS, Vajdic CM, Morton LM, et al. Medical history, lifestyle, family history, and occupational risk factors for follicular lymphoma: the InterLymph Non-Hodgkin Lymphoma Subtypes Project. J Natl Cancer Inst Monogr. 2014;2014(48):26-40. doi:10.1093/jncimonographs/lgu006

32. Perrotta C, Kleefeld S, Staines A, et al. Multiple myeloma and occupation: a pooled analysis by the International Multiple Myeloma Consortium. Cancer Epidemiol. 2013;37(3):300-305. doi:10.1016/j.canep.2013.01.008

33. Maheswaran R, Morley N. Incidence, socioeconomic deprivation, volume-outcome and survival in adult patients with acute lymphoblastic leukaemia in England. BMC Cancer. 2018;18(1):25. doi:10.1186/s12885-017-3975-0

34. Orsi L, Rudant J, Ajrouche R, et al. Parental smoking, maternal alcohol, coffee and tea consumption during pregnancy, and childhood acute leukemia: the ESTELLE study. Cancer Causes Control. 2015;26(7):1003-1017. doi:10.1007/s10552-015-0593-5

\section{CONFLICTS OF INTEREST}

The authors have completed and submitted the ICMJE Form for Disclosure of Potential Conflicts of Interest and none was reported.

FUNDING

There was no source of funding for this research.

ETHICAL APPROVAL AND INFORMED CONSENT

Ethical approval and informed consent were not required as the data used were from existing databases.

PROVENANCE AND PEER REVIEW

Not commissioned; externally peer reviewed. 